\title{
Modeling of the Limiting Digging Force of Hydraulic Excavator Based on Resistance Characteristics
}

\author{
Zhigui REN*, Junli WANG**, Zhihong ZOU***, Yanyan WANG*, Haojie ZHU* \\ *School of Mechanical Engineering, Shaanxi University of Technology, Hanzhong Shaanxi 723001, China, \\ E-mail:zhiguiren@163.com; wangyanyanustb@163.com; zhuhj1995@163.com \\ **Shaanxi Key Laboratory of Industrial Automation, Shaanxi University of Technology, Hanzhong Shaanxi 723001, China, \\ E-mail:wjl503@126.com \\ ***State Key Laboratory of Mechanic Transmission, Chongqing University, Chongqing, 400030, China, \\ E-mail: zouzhihong0507@163.com
}

crossref http://dx.doi.org/10.5755/j01.mech.25.5.22805

\section{Introduction}

The hydraulic excavator has been widely applied in many fields such as mining, engineering construction, water conservation, and agriculture [1]. Some actions are very common in the earthmoving task, mainly including digging, rotating, soil unloading, resetting. Among these actions, the digging process of the excavator is the most basic and complex. To investigate the digging process, based on experimental results and theoretical analysis, existing researches had established many models for soil-tool interaction [2-9]. Bhaveshkumar et al. [8] reviewed these models and classified them into three different groups as: 1 . for resistive force and trajectory planning; 2 . for soil properties; 3 . for soil failure. In connection with a two-dimensional cutting edge, Osman [5] proposed dividing the cutting resistance to two parts based on whether the effects of the soil cohesion forces and additional loads are taken into account and established mathematical expressions for the cutting force and its horizontal component forces under the premise of taking into account the logarithmic failure surface. Swick et al. [6] added the consideration of factors such as the viscosity and cohesive forces of the soil and further improved the cutting force model. In connection with a three-dimensional bucket-shaped device, Alekseva et al. [7] proposed the empirical formulas and equations of digging resistance that are broadly applied in terms of excavator design and automation.

Comprehensively taking various soil-tool interaction models into account, some researchers established different models for theoretical digging forces [10-16]. Generally, they include the theoretical digging force for cylinder, and the theoretical digging force for complete machine (TDFCM). In calculation models of theoretical digging forces for cylinder, Park [11] thought that each ram force is statically combined together to determine the end-effect forces at a bucket. He regardless the working device is a whole in which the total force is not the simple sum of each component. Flores et al. [12] calculated the maximal force using kinematical transformers and linear optimization method. This method ignored some constraints (overall stability, lock pressure and so on) which were considered in TDFCM.

In calculation models for theoretical digging forces, TDFCM is the most representative. It is a key index in the estimation of the excavator performance. Besides, it also acts as one of the performance parameters attracting the most attention to designers and users [13]. TDFCM serves as the maximum digging resistance that can be overcome by the excavator in the practical digging process [14-15]. According to the definition, under the same digging attitude the TDFCM is undoubtedly larger or at least equal to the digging resistance. However, the findings in many tests show that the TDFCM under some digging attitudes are smaller than the digging resistance in actual measurement (Instability occurs in the attitude that should be stable according to the TDFCM calculation method). So, it can be inferred that the maximum digging resistance which is able to be overcome by the excavator under some digging attitudes cannot be acquired accurately according to the TDFCM calculation method.

To solve the problem abovementioned, this research established a limiting digging force (LDF) model. In this model, the tangential force, normal force, and bending moment was taken into account simultaneously.

\section{Resistance characteristics of hydraulic excavator}

The aim at the theoretical digging force calculation is to obtain the maximum digging resistance the excavator can surmount in the practical digging process. The corresponding calculation results are expected to reflect the actual digging ability of the excavator. Hence, it is necessary that the calculation model for the theoretical digging force is in agreement with the practical digging process. And it can show the excavator's actual capacity to deal with the digging resistance in the digging process. Therefore, to establish the calculation model for the theoretical digging force, it is an indispensable step to investigate the real characteristics of the digging resistance.

\subsection{The composition of the digging resistance system}

As shown in Fig. 1, a, Hemami et al. [9] divided the digging resistance into six parts. In the figure, $f_{1}$ to $f_{6}$ correspond to the force to compensate for the weight of the loaded soil, the compacting resistance of unloaded soil, the friction force, the cutting resistance, the inertial force (for the loaded soil), and the force to move the empty bucket. This method classifies the complex system of the digging resistance into six force with different properties; although it helps to reveal the soil-tool interaction mechanism, the magnitude of the force is difficult to quantify. 
As shown in Fig. 1, b, to acquire the digging resistance accurately, according to the composition principle of coplanar forces, the digging resistance system $\left(f_{1} \sim f_{6}\right)$ is composed as the tangential resistance $F_{t}$ the normal resistance $F_{n}$, and the resistance moment $T_{r}$.

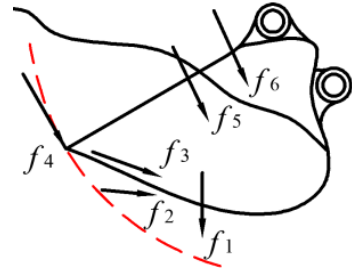

a

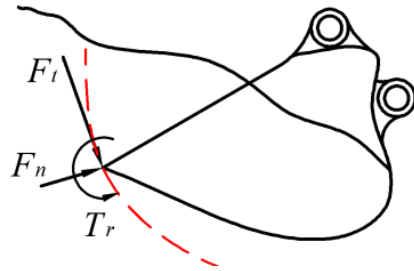

b
Fig. 1 Digging resistance system: (a) Classification; (b) Combination

2.2. The resistance coefficient and the resistance moment coefficient

The results in repeated digging resistance tests indicate that the normal resistance $F_{n}$ and the resistance moment $T_{r}$ show smaller effect than the tangential resistance $F_{t}$. Also, there is certain relation among $F_{n}, T_{r}$, and $F_{t}$ [17-18]. The ratio of $F_{n}$ to $F_{t}$ is termed as the resistance coefficient denoted by $\varepsilon$, while the ratio of $T_{r}$ to $F_{t}$ is called as the resistance moment resistance defined by $\delta$. Based on extensive experimental data for a $35 t$ hydraulic excavator with backhoe attachment, the corresponding histograms of the probability distribution for $\varepsilon$ and $\delta$ are displayed respectively in Figs. 2 and 3.

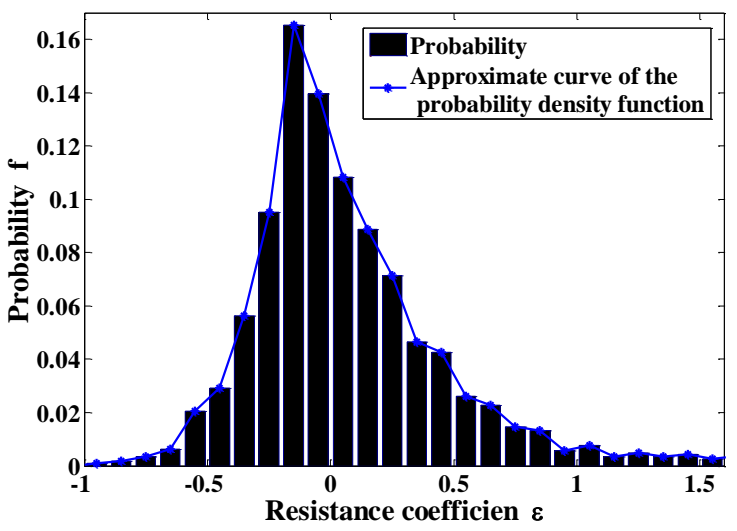

Fig. 2 Resistance coefficient $\varepsilon$

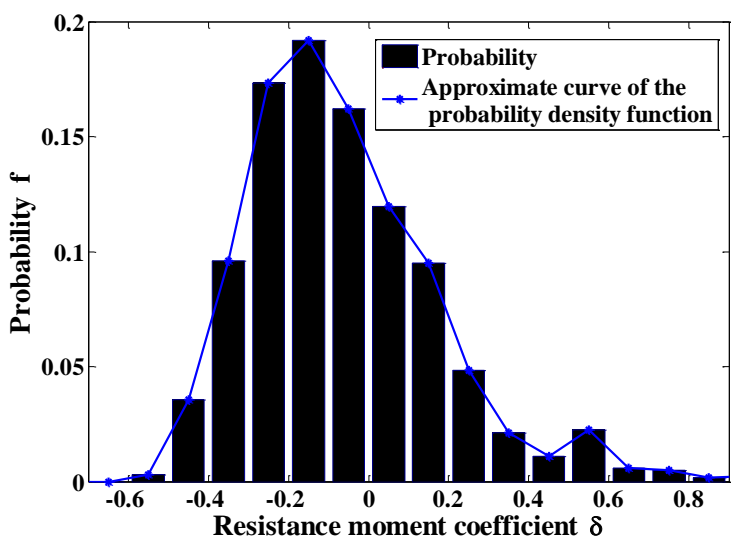

Fig. 3 Resistance moment coefficient $\delta$
The values of $\varepsilon$ and $\delta$ were not constant arguments but in a large area. And the histograms demonstrate that at most moments, values of $\varepsilon$ and $\delta$ were concentrated in certain ranges. Specifically, the values of the $\varepsilon$ are mainly $(81.5 \%)$ present in the range $-0.4 \sim 0.5$, while those of the $\delta$ are largely $(83.8 \%$ ) present in the range $-0.4 \sim 0.2$. In this way, the main value ranges of $\varepsilon$ and $\delta$ are obtained. On this basis, when the tangential resistance $F_{t}$ is also known, the possible value ranges of the normal resistance $F_{n}$ and the resistance moment $T_{r}$ can be thereby acquired.

\section{Calculation model for the LDF}

The TDFCM calculation method is based on the analysis method of the digging resistance in the soil-tool interaction models. That is, it only takes the tangential force acting on the bucket tooth tip into account, while the normal force and the bending moment are simplified or even neglected [16]. But in the real digging process, there are both the normal resistance and the resistance moment. Besides this, $\varepsilon$ and $\delta$ show continuous variation with time. Therefore, obviously, ignoring the normal force (or only simply set to one fifth of the tangential force) and the moment, the TDFCM obtained is not consistent with the practical digging process, let alone to accurately reflect the limiting digging ability of excavator. Hence, a new model reflecting the ultimate digging ability is needed.

Based on the resistance characteristics, the LDF can be defined as: With the tangential force, normal force, and bending moment simultaneously taken into consideration, the maximum applied force on the cutting edge of the bucket can be generated under the independent operation of the bucket cylinder or the arm cylinder.

\subsection{Modeling of the LDF}

The LDF of the excavator is equivalent to the maximum digging resistance that can be surmounted by the excavator. So, if we can acquire the maximum digging resistance, then, the LDF is also obtained. In the digging process, the excavator is applied by the gravity, the thrust of the cylinder, and the digging resistance simultaneously. At the same time, it achieves the dynamic equilibrium. In this paper, it is assumed that the excavator is approximately taken as the fully symmetrical structure and the force analysis on the plane of symmetry is exhibited in Fig. 4. In the figure, hollow circles denote articulated points among members; solid circles represent the centers of members' gravities; $S$ and $T$ refer to the positions of the excavator's backward line and forward line respectively. $\mathrm{J}$ denotes the position of the cutting edge on the bucket; $F_{t}, F_{n}$, and $T_{r}$ respectively represent the tangential resistance, the normal resistance, and the resistance moment; $F_{b o}, F_{a}$, and $F_{b u}$ denote the thrust of the boom cylinder, the thrust of the arm cylinder, and the thrust of the bucket cylinder respectively.

Under a certain digging attitude, the LDF of the hydraulic excavator is determined by the hydraulic pressure limit, the tipping limit, and the slipping limit. All these limiting conditions are called by a joint name complete machine limiting conditions of the LDF. For example, for the independent bucket digging, the limiting conditions include:

1. the limiting thrust of the bucket cylinder; 2 . the limiting lock force of the cavity without the rod of the arm cylinder; 
3. the limiting lock force of the cavity with the rod of the arm cylinder; 4. the limiting lock force of the cavity without the rod of the boom cylinder; 5. the limiting lock force of the cavity with the rod of the boom cylinder; 6 . the forward tipping of the complete machine; 7 . the backward tipping of the complete machine; 8 . the adhesion conditions of the complete machine. The LDF for bucket digging must satisfy all the 8 conditions simultaneously and for each condition, there is a corresponding inequality. Under a given digging attitude and limiting conditions, only the digging resistance is unknown in each inequality. By calculating the set of limiting equalities, the maximum digging resistances are acquired: respectively, $F_{1}, F_{2}, F_{3}, F_{4}, F_{5}, F_{6}, F_{7}$ and $F_{8}$. Setting $F_{\max }=\min \left(F_{1}, F_{2}, F_{3}, F_{4}, F_{5}, F_{6}, F_{7}, F_{8}\right)$, then $F_{\max }$ is exactly the LDF that satisfies all the limiting conditions and can be applied by the hydraulic excavator under a given attitude.

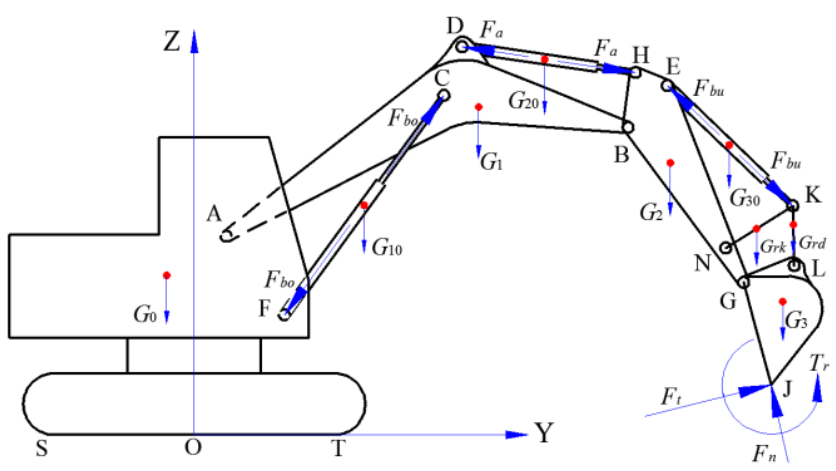

Fig. 4 The force diagram of the excavator

In the TDFCM model, the digging resistance is simplified as the tangential resistance $F_{t}$, that is, only one unknown quantity is present in the digging resistance. But, in the LDF model, there are 3 independent unknown quantities $\left(F_{t}, F_{n}, T_{r}\right)$ in the digging resistance. This is the root of the difference between the TDFCM model and the LDF model in the derivation and the solution of the limiting conditional inequalities.

For example, in bucket digging, this difference finds its expression in the inequality corresponding to the first limiting condition of the LDF. This limiting condition is corresponding to the limiting thrust of the bucket cylinder and it is used to overcome the digging resistance (the counter force of the LDF). This can be reflected directly in the fact that the bucket tends to conduct the clockwise rotation all along around the articulated point $G$ (The moment in counterclockwise rotation is set to be positive and otherwise, the moment is negative). To realize it, we must ensure that the moment $M_{G}$ for the force system of the bucket versus the point $\mathrm{G}$ cannot exceed 0 , that is, $M_{G} \leq 0$.

In the TDFCM model only taking the tangential force into account, there is:

$$
M_{G}=\overrightarrow{G L} \overrightarrow{F_{K L}}+\overrightarrow{G g_{3}} \overrightarrow{G_{3}}+\overrightarrow{G J} \overrightarrow{F_{t}} \leq 0,
$$

where: $F_{K L}$ denotes the force that arises from the limiting thrust of the bucket cylinder $F_{b u}$ and then acts on the articulated point $\mathrm{L}$ on the bucket via the connecting rod $\mathrm{KL}$; $F_{K L}=f\left(F_{b u}\right) \cdot \overrightarrow{G g_{3}}$ represents the vector with its direction from the articulated point $\mathrm{G}$ on the bucket to the center of the bucket's gravity $g_{3}$. And the $G_{3}$ refers to the bucket's gravity.

In the LDF model taking the tangential force, the normal force, and the moment simultaneously into consideration, the following relation is acquired:

$$
M_{G}=\overrightarrow{G L} \overrightarrow{F_{K L}}+\overrightarrow{G g_{3}} \overrightarrow{G_{3}}+\overrightarrow{G J} \overrightarrow{F_{t}}+\overrightarrow{G J} \overrightarrow{F_{n}}+T_{r} \leq 0 .
$$

In the Eq. (1), only the magnitude of the tangential force $F_{t}$ is an independent unknown quantity. By directly calculating the Eq. (1), the maximum $F_{t}$ satisfying this inequality can be obtained. In the Eq. (2), there are three independent unknown quantities: $F_{t}, F_{n}$, and $T_{r}$. According to only one inequality, we cannot acquire three unknown quantities. Similarly, when the $F_{t}, F_{n}$, and $T_{r}$ are considered simultaneously, the inequalities corresponding to all the other limiting conditions cannot be calculated either. Therefore, we must ensure that all the three components of the digging resistance are taken into account. On this basis, it is also required that the three variables in the digging resistance should be transformed into one independent unknown quantity.

To achieve this aim, we should analyze and then utilize the relation among the normal resistance $F_{n}$, the resistance moment $T_{r}$, and the tangential resistance $F_{t}$ in the practical digging process. For a long time, the tangential force has been the focus of designers and users. Therefore, it is taken as the independent unknown quantity in this research and the other two (the normal force and the moment) act as the functions of the tangential force. In this way, three variables renders into only one independent unknown quantity. The statistical analysis on the $\varepsilon$ and $\delta$ in the last section indicates that with the change of digging conditions in the whole digging process, $\varepsilon$ and $\delta$ vary continuously, but it is far from easy to reveal their variation law. Therefore, it is impossible to establish the precise functional relationship among $F_{n}, T_{r}$, and $F_{t}$. But based on the statistical results, values of $\varepsilon$ and $\delta$ are concentrated in the significant main ranges.

The tangential resistance $F_{t}$ is set as an independent unknown quantity. The resistance moment $T_{r}=\delta F_{t}$ and the normal resistance $\overrightarrow{F_{n}}=\varepsilon[T] \overrightarrow{F_{t}}$. Where $T$ denotes the rotation matrix from the tangential resistance to the normal resistance, $[T]=[0,-1,0 ; 1,0,0 ; 0,0,1]$.

Then, the rotation matrix above mentioned is substituted into the Eq. (2) and the result obtained is demonstrated as follow:

$$
M_{G}=\overrightarrow{G L} \overrightarrow{F_{K L}}+\overrightarrow{G g_{3}} \overrightarrow{G_{3}}+\overrightarrow{G J}(I+\varepsilon[T]) \overrightarrow{F_{t}}+\delta F_{t} \leq 0
$$

where: $I$ is denotes the unit matrix.

When $\varepsilon$ and $\delta$ are known, the magnitude of the tangential resistance $F_{t}$ is the only one independent variable in the Eq. (3). By calculating the Eq. (3), the maximum digging resistance $F_{t}$ that satisfies the conditions of the limiting thrust of the bucket cylinder is obtained. Similarly, according to other limiting conditional inequalities, finally, 
we can acquired the maximum digging resistance satisfying all the limiting conditions, namely the LDF $F_{\max }$.

\subsection{The calculation of the LDF}

When $\varepsilon$ and $\delta$ take a set of values in their possible ranges, according to the limiting inequality set, the corresponding maximum digging resistance overcome by the excavator (that is, the possible LDF $F_{\text {maxi }}$ ) is acquired. When $\varepsilon$ and $\delta$ take another set of values, another one possible LDF $F_{\operatorname{maxj}}$ is then calculated. In this way, at last, we can obtain all the possible limiting digging forces among which the maximum value is exactly the LDF $F_{\max }$ under a certain digging posture of the excavator.

In section 2 of this paper, the main value ranges of the digging resistance coefficient $\varepsilon$ and the resistance moment coefficient $\delta$ are obtained by conducting repeated digging experiments on a $35 t$ hydraulic excavator with backhoe attachment. One given digging posture of this excavator working device is taken as example. $\varepsilon$ and $\delta$ take their values in their main ranges. According to the LDF model mentioned in section 3.1, for each given value set of $\varepsilon$ and $\delta$, the corresponding possible LDF in bucket digging is acquired (Fig. 5). The given digging posture is set as follows: the angle between the boom and the shutdown surface $\theta_{1}=10^{\circ}$; the angle between the arm and the boom $\theta_{2}=90^{\circ}$; the angle between the bucket and the arm $\theta_{3}=120^{\circ}$.

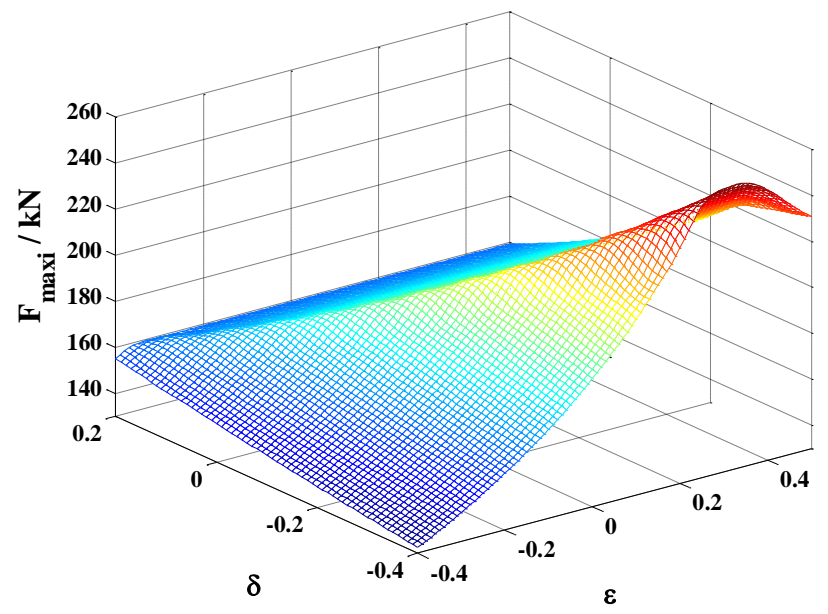

Fig. 5 The possible LDF $F_{\text {maxi }}$

As illustrated in Fig. 5, under the same digging posture, for diverse value sets of $\varepsilon$ and $\delta$, the corresponding possible LDF $F_{\text {maxi }}$ is also different. Based on the calculation results, when both $\varepsilon$ and $\delta$ are set to -0.4 , the minimum calculated value of the corresponding possible LDF is achieved, reaching to $F_{\text {maxi }}=132.6 \mathrm{kN}$. When $\varepsilon=0.35$ and $\delta=-0.4$, the maximum calculated value of the LDF is calculated: $F_{\text {maxa }}=252.9 \mathrm{kN}$. In this way, under this digging posture of the excavator, the LDF in bucket digging is thereby acquired: $F_{\max }=252.9 \mathrm{kN}$.

In Fig. 5, with the variation of $\varepsilon$ and $\delta$ in their possible value ranges, the curved surface of the corresponding possible LDF varies gradually and smoothly. So, it can be inferred that for two approximate value sets of $\varepsilon$ and $\delta$, the corresponding limiting digging forces obtained are also similar. Thus, to show LDF in all the possible value sets of $\varepsilon$ and $\delta$, we only need to calculate the LDF corresponding to limited value sets of $\varepsilon$ and $\delta$. Hence, as displayed in Fig. 6, $\varepsilon$ and $\delta$ are required to take their values in their possible value ranges at a certain step ( 0.05 in this paper). For each set of values, according to the inequality set resulting from limiting conditions, one corresponding possible LDF is thereby acquired. By repeating the above process, we can obtain all the possible limiting digging forces. The maximum one among them is exactly the LDF under the given digging posture and digging pattern of the excavator.

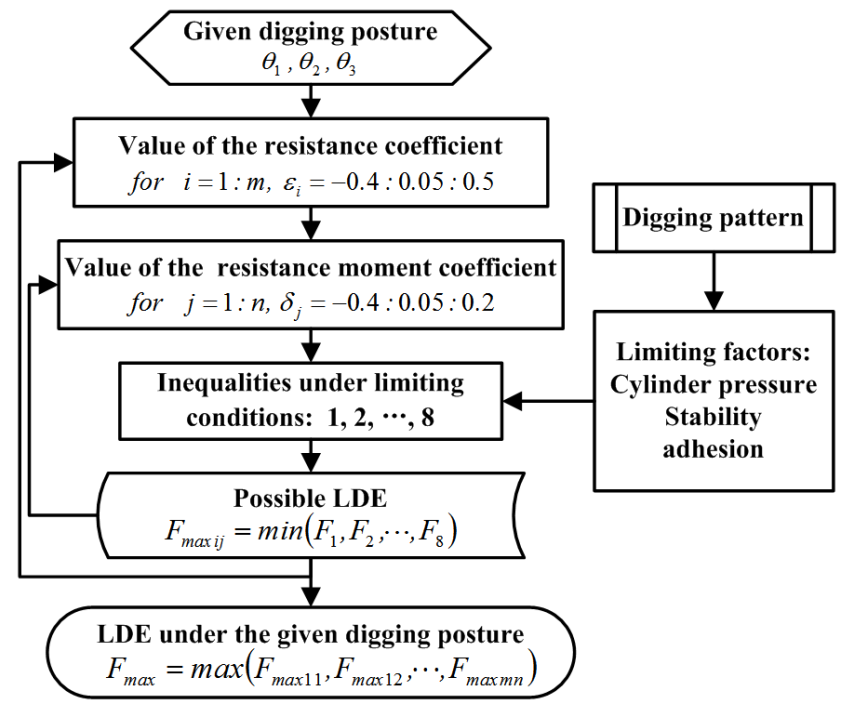

Fig. 6 The calculation process of the LDF

In the TDFCM model, the normal resistance and the resistance moment are ignored. The result obtained in this situation is similar to the possible LDF when both $\varepsilon$ and $\delta$ are set to 0 in the calculation process of the LDF. Since the LDF is the maximum value among all possible limiting digging forces, the LDF is definitely larger than TDFCM. Under the given digging posture $\left(\theta_{1}=10^{\circ}, \theta_{2}=90^{\circ}\right.$, $\theta_{3}=120^{\circ}$ ) of the excavator, when both $\varepsilon$ and $\delta$ are set to 0 , the corresponding TDFCM $F=181.7 \mathrm{kN}$. In this case, the $\operatorname{LDF}\left(F_{\max }=252.9 \mathrm{kN}\right)$ is $39.2 \%$ larger than the TDFCM.

\section{Comparative analysis based on experimental results}

In many digging experiments on the $35 t$ hydraulic excavator with backhoe attachment, this research tested for both working device postures and the cylinder pressure synchronously. At the same time, the digging resistances under all posture are also acquired. Fig. 7, a, exhibits the distribution of the actual digging resistances under all digging postures (digging posture determined the spatial position of cutting edge).

The TDFCM model and the LDF model were adopted respectively to acquire the theoretical digging force under the practical digging postures (those recorded in the experiments) of the excavator. The corresponding results are displayed in Fig. 7, b, and c.

The theoretical digging force is the maximum digging force provided by the excavator in a given posture, that is, the maximum digging resistance overcome by the excavator. So, the theoretical digging force is undoubtedly larger or at least equal to the actual digging resistance. But, the 
analysis on the calculation results indicates that under only $78.2 \%$ of digging postures, TDFCM is larger than the actual digging resistance. In comparison, under all digging postures, the LDF is larger than the TDFCM and also larger than the actual digging resistance.

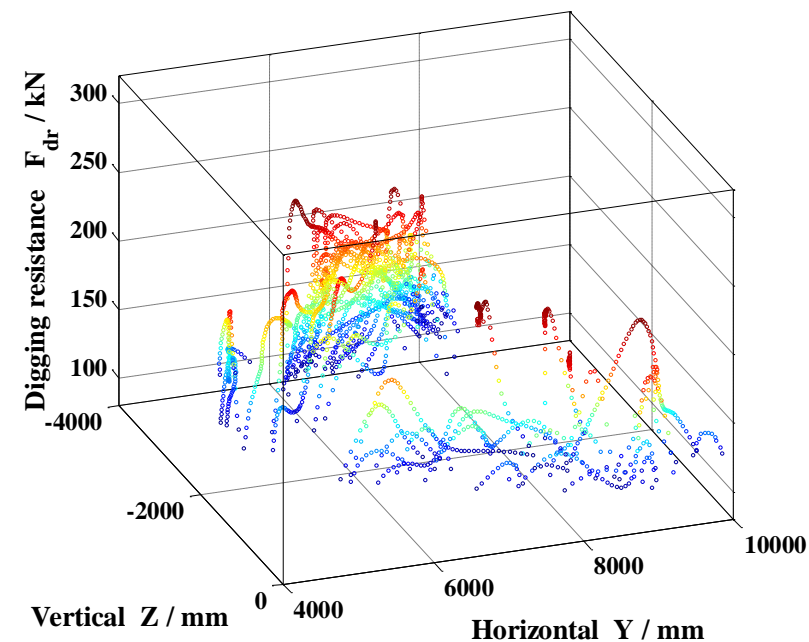

a

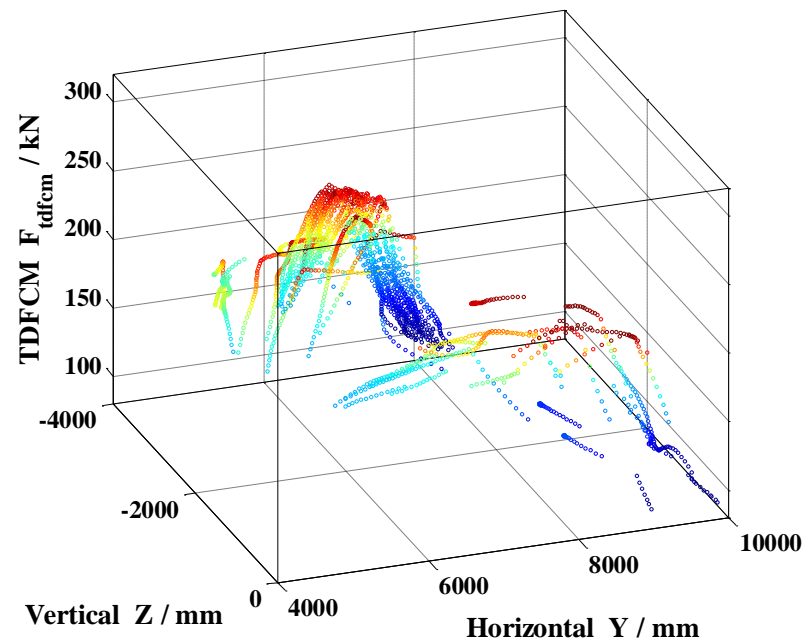

b

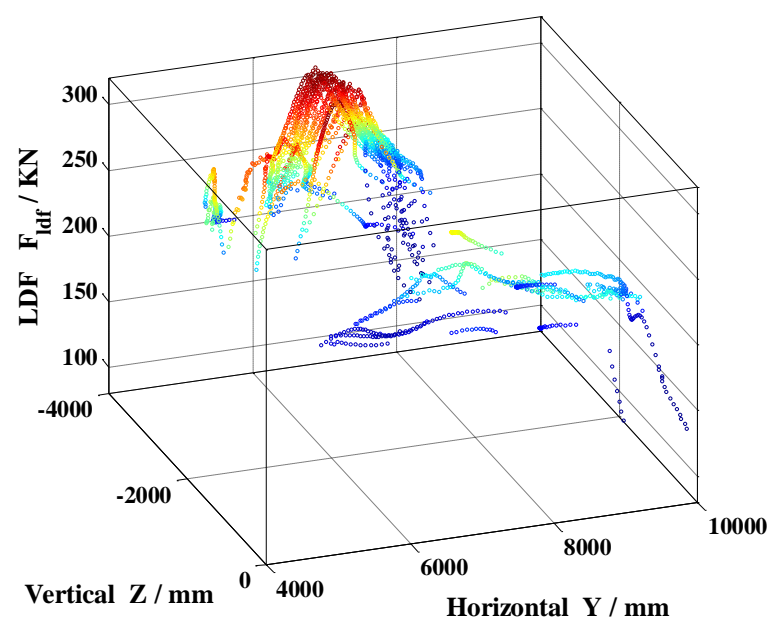

c

Fig. 7 The distribution of (a) actual digging resistances; (b) TDFCM; (c) LDF

\section{Conclusion}

The accurate calculation of the theoretical digging force shows many applications: not only in the optimal design of the excavator and the evaluation of the excavator's digging performance, but also in trajectory planning and control automation. In TDFCM model the normal resistance and resistance moment are simplified and ignored. Based on the resistance characteristics, the LDF model is established in this paper, simultaneously taking the tangential force, normal force, and the bending moment into consideration.

Taking the digging resistance by testing for a $35 t$ hydraulic excavator with backhoe attachment as the standard, this research compares the calculation results of the TDFCM model with those of the LDF model proposed in this paper. The calculation results show that: according to TDFCM model, under $22.8 \%$ of digging postures, the corresponding theoretical digging force is smaller than the actual digging resistance. This indicates that under limited working conditions, the TDFCM calculation method can reveal the digging ability of the excavator to some degree. According to LDF model, under all digging postures, the corresponding theoretical digging force is larger than or equal to both the TDFCM and the actual digging resistance. In this way, it is verified that the LDF is never smaller than the TDFCM. This is also consistent with the fact that the theoretical digging force is larger than or at least equal to the actual digging resistance.

All these findings indicate that the LDF model takes more factors into consideration and is consistent with the practical digging process. Thereby, this model can accurately calculate the LDF of the excavator under any posture. The accurate calculation of the LDF can lay reliable foundation for the mechanism optimization, structural strength design, trajectory planning, and control automation of the excavator.

\section{Acknowledgements}

This work was funded by National Natural Science Foundation of China (No: 51605270), the Natural Science Research Project of Shaanxi Province (No: 2019JQ-884) and the Shaanxi Provincial Department of Education Scientific Re-search Project (No: 18JK0150).

\section{References}

1. Coetzee, C. J.; Els, D. N. J. 2009. The numerical modeling of excavator bucket filling using DEM, Journal of Terramechanics 46(5): 217-227. https://doi.org/10.1016/j.jterra.2009.05.003.

2. Zhang, L. B.; Cai, Z. X.; Liu, H. F. 2018. A novel approach for simulation of soil-tool interaction based on an arbitrary Lagrangian-Eulerian description, Soil and Tillage Research 178: 41-49. https://doi.org/10.1016/j.still.2017.12.011.

3. Blouin, S.; Hemami, A.; Lipsett, M. 2001.Review of resistive force models for earthmoving processes, Journal of Aerospace Engineering 14(3): 102-111. https://doi.org/10.1061/(ASCE)08931321(2001)14:3(102).

4. Jiang, M. J.; Xi, B. L.; Arroyo, M.; \& RodriguezDono, A. 2017. DEM simulation of soil-tool interaction under extraterrestrial environmental effects, Journal of Terramechanics 71: 1-13. https://doi.org/10.1016/j.jterra.2017.01.002.

5. Osman, M. S. 1964. The mechanics of soil cutting 
blades, J. Agric. Engng Res 9(4): 313-328. https://doi.org/10.1016/0022-4898(65)90115-1.

6. Swick, W. C.; Perumpral, J. V. 1988. A model for predicting soil-tool interaction. Journal of Terramechanics 25(1): 43-56. https://doi.org/10.1016/0022-4898(88)90061-4.

7. Alekseeva, T. V.; Artem'ev, K. A.; Bromberg, A. A.; Voitsekhovskii, R. I.; Ul'yanov, N. A. 1985. Machines for earthmoving work, theory and calculation. (Balkema, Rotterdam, The Netherlands).

8. Bhaveshkumar, P. P.; Prajapati, J. M. 2011. Soil-tool interaction as a review for digging operation of mini hydraulic excavator, International Journal of Engineering Science and Technology 3(2): 894-901.

9. Hemami, A.; Goulet, S.; Aubertin, M. 1994. Resistance of particulate media to excavation: Application to bucket loading, International Journal of Surface Mining and Reclamation 8(3): 125-129. https://doi.org/10.1080/09208119408964772.

10. Chen, S. J.; Rong, H. J.; Ji, M. Z.; Du, B.; Xu, L. P. 2007. Calculation of theoretical digging force under combined work condition for hydraulic excavator with backhoe attachment and its application, Construction and Equipment 38(4): 39-43. https://doi.org/10.3969/j.issn.1000-1212.2007.04.013.

11. Park, B. 2002. Development of a Virtual Reality Excavator Simulator: a Mathematical Model of Excavator Digging and a Calculation Methodology, Blacksburg, Virginia: Virginia Polytechnic Institute \& State University.

12. Flores, F. G.; Kecskemethy, A.; pottker, A. 2007. Workspace analysis and maximal force calculation of a face-shovel excvator using kinematical transformers, 12th IFToMM Word Congress, 12th World Congress in Mechanism and Machine Science, Besancon, France: $18-21$.

https://pdfs.semanticscholar.org/2494/4e58ef0f8af4ffb6 5b a700ad2465ed1624a9.pdf.

13. Huang, B.; He, Q. H.; He, J. L.; Wang, B. Z.; Jiang, R. B. 2009. Research on drawing mine map of backhoe hydraulic excavator, Transactions of the Chinese Society for Agricultural Machinery 40(9): 26-31. https://doi.org/10.1360/972009-1549.

14. Shi, Q. L.; Lian, J. Y.; Lin, M. Y. 2007. The confirmation of the theoretical maximum digging force of excavator, Journal of Taiyuan university of science and technology 28(1): 32-35. https://doi.org/10.3969/j.issn.1673-2057.2007.01.007.

15. Chang, L.; Zhang, J. H. 2011. Excavating force analysis and calculation of dipper handle, 2011 International Conference on Consumer Electronics, Communications and Networks 14: 102-111. https://doi.org/10.1109/CECNET.2011.5768595.

16. Chen, J.; Li, W. B.; Zhang, S. Q.; Zhong, C. J. 2008. Experimental research on digging resistance for large hydraulic face-shovel, China Mechanical Engineering 19(5): 518-521.
https://doi.org/10.3321/j.issn:1004-132X.2008.05.004.

17. Ren, Z. G.; Chen, J.; Wang, S. C.; Pang, X. P. 2015. Resistance characteristics of hydraulic excavator, Journal of Northeastern University 36(4), 551-556. https://doi.org/10.3969/j.issn.1005-3026.2015.04.02.

18. Chen, J.; Zou, Z. H.; Pang, X. P. 2018. Digging performance characterization for hydraulic excavator considering uncertainty during digging operation, Proceedings of the Institution of Mechanical Engineers, Part C: Journal of Mechanical Engineering Science 232(5), 857871.

https://doi.org/10.1177/0954406217692843.

Z. Ren, J. Wang, Z. Zou, Y. Wang, H. Zhu

MODELING OF THE LIMITING DIGGING FORCE OF HYDRAULIC EXCAVATOR BASED ON RESISTANCE CHARACTERISTICS

S u m m a r y

Based on the resistance characteristics, a model of theoretical digging force was proposed in this paper, taking the tangential force, the normal force, and the bending moment into account simultaneously. Utilizing the relation among the normal resistance, the resistance moment, and the tangential resistance in practical digging process, three independent unknown quantities are transformed into only one variable. Afterwards, according to different digging patterns and complete machine limiting conditions, this research derived the constraint inequalities of the limiting digging force (LDF) and established the calculation models for LDF. Then, based on the value distribution laws of the digging resistance coefficient and the resistance moment coefficient, the calculation process and corresponding method of LDF under a given digging posture were obtained. Taking the digging resistance obtained by testing for $35 t$ hydraulic excavator with backhoe attachment as the reference, this paper compared the calculation results of the theoretical digging force for complete machine with those of the LDF model proposed in this research. The comparative results indicate that the LDF is consistent with the fact that the theoretical digging force is larger than or at least equal to the actual digging resistance. So, the LDF can exactly show the real limiting digging ability of the excavator more accurately. In this way, it can provide basis for mechanism optimization, structural strength design, trajectory planning, and control automation of the excavator.

Keywords: hydraulic excavator, digging resistance, theoretical digging force, limiting digging force.

Received February 20, 2019 Accepted October 14, 2019 